\title{
A NOTE ON THE STRONG CONVERGENCE OF PARTIAL SUMS WITH RESPECT TO VILENKIN SYSTEM
}

\author{
G. TUTBERIDZE
}

\begin{abstract}
In this paper we investigate some strong convergence theorems for partial sums with respect to Vilenkin system.
\end{abstract}

2010 Mathematics Subject Classification. 42C10.

Key words and phrases: Vilenkin system, partial sums, Fejér means, Hardy space, strong convergence.

\section{INTRODUCTION}

It is well-known (for details see e.g. [8] and [14]) that the Vilenkin system does not form a basis in the space $L_{1}\left(G_{m}\right)$. Moreover, there is a function in the Hardy space $H_{1}\left(G_{m}\right)$, (for details see [12, 13, 21, 22]) such that the partial sums of $f$ are not bounded in $L_{1}$-norm. However, (see e.g. [2, 23]) the subsequence $S_{M_{n}}$ of partial sums are bounded from the Hardy space $H_{1}\left(G_{m}\right)$ to the Lebesgue space $L_{1}\left(G_{m}\right)$ :

$$
\left\|S_{M_{k}} f\right\|_{H_{1}} \leq c\|f\|_{H_{1}} \quad(k \in \mathbb{N}) .
$$

Moreover, in Gát [7] (see also Simon [18, 19]) it was proved the following strong convergence result for all $f \in H_{1}$ :

$$
\lim _{n \rightarrow \infty} \frac{1}{\log n} \sum_{k=1}^{n} \frac{\left\|S_{k} f-f\right\|_{1}}{k}=0
$$

where $S_{k} f$ denotes the $k$-th partial sum of the Vilenkin-Fourier series of $f$.

It follows that there exists an absolute constant $c$, such that

$$
\frac{1}{\log n} \sum_{k=1}^{n} \frac{\left\|S_{k} f\right\|_{1}}{k} \leq c\|f\|_{H_{1}} \quad(n=2,3 \ldots)
$$

and

$$
\lim _{n \rightarrow \infty} \frac{1}{\log n} \sum_{k=1}^{n} \frac{\left\|S_{k} f\right\|_{1}}{k}=\|f\|_{H_{1}}
$$

for all $f \in H_{1}$.

Analogical result for the trigonometric system was proved by Smith [20], and for the Walsh-Paley system by Simon [17].

The research was supported by a Swedish Institute scholarship and by Shota Rustaveli National Science Foundation grant YS15-2.1.1-47. 
If the partial sums of Vilenkin-Fourier series was bounded from $H_{1}$ to $L_{1}$ we also would have:

$$
\sup _{n \in \mathbb{N}_{+}} \frac{1}{n} \sum_{m=1}^{n}\left\|S_{m} f\right\|_{1} \leq c\|f\|_{H_{1}},
$$

but as it was presented above the boundednes of the partial sums does not hold from $H_{1}$ to $L_{1}$, However, we have inequality (2).

On the other hand, in the one-dimensional case, Fujji [6] and Simon [16] proved that maximal operator Fejér means are bounded from $H_{1}$ to $L_{1}$, that is

$$
\sup _{n \in \mathbb{N}_{+}}\left\|\frac{1}{n} \sum_{m=1}^{n} S_{m} f\right\|_{1}<c\|f\|_{H_{1}} .
$$

So, natural question has arised that if inequality (3) holds true, which would be generalization of inequality (4) or is we have negative answer on this problem.

In this paper we prove that there exists a function $f \in H_{1}$ such that

$$
\sup _{n \in \mathbb{N}_{+}} \frac{1}{n} \sum_{m=1}^{n}\left\|S_{m} f\right\|_{1}=\infty .
$$

This paper is organized as follows: in order not to disturb our discussions later on some definitions and notations are presented in Section 2. For the proofs of the main results we need some auxiliary Lemmas. These results are presented in Section 3. The formulation and detailed proof of main results can be found in Section 4 .

\section{Definitions and Notations}

Let $\mathbb{N}_{+}$denote the set of the positive integers, $\mathbb{N}:=\mathbb{N}_{+} \cup\{0\}$.

Let $m:=\left(m_{0}, m_{1}, \ldots\right)$ denote a sequence of positive integers not less than 2 .

Denote by

$$
Z_{m_{k}}:=\left\{0,1, \ldots, m_{k}-1\right\}
$$

the additive group of integers modulo $m_{k}$.

Define the group $G_{m}$ as the complete direct product of the group $Z_{m_{j}}$ with the product of the discrete topologies of $Z_{m_{j}}$ 's.

The direct product $\mu$ of the measures

$$
\mu_{k}(\{j\}):=1 / m_{k} \quad\left(j \in Z_{m_{k}}\right)
$$

is the Haar measure on $G_{m}$ with $\mu\left(G_{m}\right)=1$.

If $\sup _{n \in \mathbb{N}} m_{n}<\infty$, then we call $G_{m}$ a bounded Vilenkin group. If the generating sequence $m$ is not bounded, then $G_{m}$ is said to be an unbounded Vilenkin group.

The elements of $G_{m}$ are represented by sequences

$$
x:=\left(x_{0}, x_{1}, \ldots, x_{k}, \ldots\right) \quad\left(x_{k} \in Z_{m_{k}}\right) .
$$


It is easy to give a base for the neighbourhood of $G_{m}$ namely

$$
\begin{aligned}
I_{0}(x) & :=G_{m}, \\
I_{n}(x) & :=\left\{y \in G_{m} \mid y_{0}=x_{0}, \ldots, y_{n-1}=x_{n-1}\right\}\left(x \in G_{m}, n \in \mathbb{N}\right)
\end{aligned}
$$

Denote $I_{n}:=I_{n}(0)$ for $n \in \mathbb{N}$ and $\overline{I_{n}}:=G_{m} \backslash I_{n}$.

Let

$$
e_{n}:=\left(0, \ldots, 0, x_{n}=1,0, \ldots\right) \in G_{m} \quad(n \in \mathbb{N}) .
$$

If we define the so-called generalized number system based on $m$ in the following way:

$$
M_{0}:=1, \quad M_{k+1}:=m_{k} M_{k}, \quad(k \in \mathbb{N})
$$

then every $n \in \mathbb{N}$ can be uniquely expressed as $n=\sum_{k=0}^{\infty} n_{j} M_{j}$ where $n_{j} \in Z_{m_{j}}(j \in \mathbb{N})$ and only a finite number of $n_{j}$ 's differ from zero. Let $|n|:=\max \left\{j \in \mathbb{N} ; n_{j} \neq 0\right\}$.

Next, we introduce on $G_{m}$ an orthonormal system, which is called the Vilenkin system.

At first define the complex valued function $r_{k}(x): G_{m} \rightarrow \mathbb{C}$, the generalized Rademacher functions as

$$
r_{k}(x):=\exp \left(2 \pi \imath x_{k} / m_{k}\right) \quad\left(\imath^{2}=-1, x \in G_{m}, k \in \mathbb{N}\right) .
$$

Now define the Vilenkin system $\psi:=\left(\psi_{n}: n \in \mathbb{N}\right)$ on $G_{m}$ as:

$$
\psi_{n}(x):=\prod_{k=0}^{\infty} r_{k}^{n_{k}}(x) \quad(n \in \mathbb{N}) .
$$

Specially, we call this system the Walsh-Paley one if $m \equiv 2$.

The norm (or quasi norm) of the space $L_{p}\left(G_{m}\right)$ is defined by

$$
\|f\|_{p}:=\left(\int_{G_{m}}|f(x)|^{p} d \mu(x)\right)^{1 / p} \quad(0<p<\infty) .
$$

The Vilenkin system is orthonormal and complete in $L_{2}\left(G_{m}\right)$ (for details see e.g. [1, 25]).

If $f \in L_{1}\left(G_{m}\right)$ we can define Fourier coefficients, partial sums of the Fourier series, Fejér means, Dirichlet kernels with respect to the Vilenkin system in the usual manner:

$$
\begin{aligned}
\widehat{f}(k):=\int_{G_{m}} f \bar{\psi}_{k} d \mu & (k \in \mathbb{N}) \\
S_{n} f:=\sum_{k=0}^{n-1} \widehat{f}(k) \psi_{k} & \left(n \in \mathbb{N}_{+}, S_{0} f:=0\right) \\
\sigma_{n} f:=\frac{1}{n} \sum_{k=0}^{n-1} S_{k} f & \left(n \in \mathbb{N}_{+}\right) \\
D_{n}:=\sum_{k=0}^{n-1} \psi_{k} & \left(n \in \mathbb{N}_{+}\right) .
\end{aligned}
$$


Recall that

$$
D_{M_{n}}(x)= \begin{cases}M_{n}, & x \in I_{n} \\ 0, & x \notin I_{n}\end{cases}
$$

and

$$
D_{s_{n} M_{n}}=D_{M_{n}} \sum_{k=0}^{s_{n}-1} \psi_{k M_{n}}=D_{M_{n}} \sum_{k=0}^{s_{n}-1} r_{n}^{k}, \quad 1 \leq s_{n} \leq m_{n}-1 .
$$

The $n$-th Lebesgue constant is defined in the following way

$$
L_{n}=\left\|D_{n}\right\|_{1} .
$$

It is well-known [25] that

$$
L_{n}=O(\log n), \quad n \rightarrow \infty .
$$

Moreover, (for unbounded Vilenkin systems it can be found in [5], for bounded Vilenkin systems see e.g. [9] and [11, 24]) there exist absolute constant $c_{1}$ and $c_{2}$ such that

$$
c_{1} \log n \leq \frac{1}{n} \sum_{k=1}^{n} L(k) \leq c_{2} \log n, \quad(n=2,3, \ldots) .
$$

The concept of the Hardy space [4] can be defined in various manners, e.g. by a maximal function

$$
f^{*}:=\sup _{n}\left|S_{M_{n}} f\right| \quad\left(f \in G_{m}\right),
$$

saying that $f$ belongs to the Hardy space if $f^{*} \in L^{1}\left(G_{m}\right)$. This definition is suitable if the sequence $m$ is bounded. In this case a good property of the space $\left\{f \in L^{1}\left(G_{m}\right): f^{*} \in L^{1}\left(G_{m}\right)\right\}$ is the atomic structure [4]. To the definition of space of Hardy type for an arbitrary $m$, first we give the concept of the atoms [16]. A set $I \subset G_{m}$ is called an interval if for some $x \in G_{m}$ and $n \in N, I$ is of the form $I=\bigcup_{k \in U} I_{n}(x, k)$, where $U$ is obtained from $Z_{m_{n}}$ by dyadic partition.

The sets $U_{1}, U_{2}, \ldots \subset Z_{m_{n}}$, are obtained by means of such a partition if

$$
\begin{gathered}
U_{1}=\left\{0, \ldots,\left[\frac{m_{n}}{2}\right]-1\right\}, \quad U_{2}=\left\{\left[\frac{m_{n}}{2}\right], \ldots, m_{n}-1\right\}, \\
U_{3}=\left\{0, \ldots,\left[\frac{\left[m_{n} / 2\right]-1}{2}\right]-1\right\}, \quad U_{4}=\left\{\left[\frac{\left.m_{n} / 2\right]-1}{2}\right], \ldots,\left[\frac{m_{n}}{2}\right]-1\right\}, \ldots
\end{gathered}
$$

etc.; [ ] denotes the entire part. We define the atoms as follows: the function $a \in L^{\infty}\left(G_{m}\right)$ is called an atom if eather $a \equiv 1$ or there exists an interval $I$ for which $\sup a \subset I,|a| \leq|I|^{-1}$ and $\int_{I} a=0$ hold. $(|I|$ denotes the Haar measure of $I)$.

Now we can define the space $H_{1}\left(G_{m}\right)$ (for details see e.g [26, 27]) as the set of all functions $f=\sum_{i=0}^{\infty} \lambda_{i} a_{i}$,where $a_{i}$ 's are atoms and for the coefficients 
$\lambda_{i}$ we have $\sum_{i=0}^{\infty}\left|\lambda_{i}\right|<\infty$. $H_{1}\left(G_{m}\right)$ is a Banach space with respect to the norm

$$
\|f\|_{H_{1}}:=\inf \sum_{k=0}^{\infty}\left|\lambda_{k}\right|<\infty .
$$

The infimum is taken over all decompositions

$$
f=\sum_{i=0}^{\infty} \lambda_{i} a_{i} .
$$

It is known [7] that $\|f\|_{H_{1}}$ is equivalent to $\left\|f^{* *}\right\|_{1}\left(f \in L^{1}\left(G_{m}\right)\right)$, where $f^{* *}(x):=\sup _{I}|I|^{-1}\left|\int_{I} f\right|,\left(x \in G_{m}, x \in I\right.$ and $I$ is interval). Since by (5)

$$
f^{*}(x)=\sup _{n \in \mathbb{N}} \frac{1}{\left|I_{n}(x)\right|}\left|\int_{I_{n}(x)} f(u) \mu(u)\right|
$$

we have $f^{*} \leq f^{* *}$ and, thus, $H\left(G_{m}\right) \subset\left\{f \in L^{1}\left(G_{m}\right): f^{*} \in L^{1}\left(G_{m}\right)\right\}$. Moreover these spaces coincide if the sequence $m$ is bounded.

\section{Main Result}

Our main result reads:

Theorem 1. a) Let $f \in H_{1}$. Then there exists an absolute constant $c$, such that

$$
\sup _{n \in \mathbb{N}} \frac{1}{n \log n} \sum_{k=1}^{n}\left\|S_{k} f\right\|_{1} \leq\|f\|_{H_{1}} .
$$

b) Let $\varphi: N_{+} \rightarrow[1, \infty)$ be a nondecreasing function satisfying the condition

$$
\varlimsup_{n \rightarrow \infty} \frac{\log n}{\varphi_{n}}=+\infty .
$$

Then there exists a function $f \in H_{1}$, such that

$$
\sup _{n \in \mathbb{N}} \frac{1}{n \varphi_{n}} \sum_{k=1}^{n}\left\|S_{k} f\right\|_{1}=\infty .
$$

Corollary 1. (see e.g. [10, 16, 18]) There exists a function $f \in H_{1}$, such that

$$
\sup _{n \in \mathbb{N}} \frac{1}{n} \sum_{k=1}^{n}\left\|S_{k} f\right\|_{1}=\infty
$$




\section{Proof of Theorem 1}

Proof. By using (17) we can conclude that

$$
\frac{1}{n \log n} \sum_{k=1}^{n}\left\|S_{k} f\right\|_{1} \leq \frac{c\|f\|_{H_{1}}}{n \log n} \sum_{k=1}^{n} \log k \leq c\|f\|_{H_{1}} .
$$

The proof of part a) is complete.

Under the condition (10) there exists an increasing sequence of the positive integers $\left\{\alpha_{k}: k \in \mathbb{N}\right\}$ such that

$$
\varlimsup_{k \rightarrow \infty} \frac{\log M_{\alpha_{k}}}{\varphi_{2 M_{\alpha_{k}}}}=+\infty
$$

and

$$
\sum_{k=0}^{\infty} \frac{\varphi_{2 M_{\alpha_{k}}}^{1 / 2}}{\log ^{1 / 2} M_{\alpha_{k}}}<c<\infty
$$

Let

$$
f=\sum_{k=1}^{\infty} \lambda_{k} a_{k}
$$

where

and

$$
a_{k}=r_{\alpha_{k}} D_{M_{\alpha_{k}}}=D_{2 M_{\alpha_{k}}}-D_{M_{\alpha_{k}}}
$$

$$
\lambda_{k}=\frac{\varphi_{2 M_{\alpha_{k}}}^{1 / 2}}{\log ^{1 / 2} M_{\alpha_{k}}} .
$$

By the definition of $H_{1}$ and (9), if we apply (11) we can conclude that $f \in H_{1}$. Moreover,

$$
\widehat{f}(j)=\left\{\begin{array}{lc}
\lambda_{k}, & j \in\left\{M_{\alpha_{k}}, \ldots, 2 M_{\alpha_{k}}-1\right\}, k \in \mathbb{N} \\
0, & j \notin \bigcup_{k=1}^{\infty}\left\{M_{\alpha_{k}}, \ldots, 2 M_{\alpha_{k}}-1\right\} .
\end{array}\right.
$$

Since

$$
D_{j+M_{\alpha_{k}}}=D_{M_{\alpha_{k}}}+\psi_{M_{\alpha_{k}}} D_{j}, \quad \text { when } j \leq M_{\alpha_{k}},
$$

if we apply (12) we obtain that

$$
\begin{aligned}
S_{j} f & =S_{M_{\alpha_{k}}} f+\sum_{v=M_{\alpha_{k}}}^{j-1} \widehat{f}(v) \psi_{v} \\
& =S_{M_{\alpha_{k}}} f+\lambda_{k} \sum_{v=M_{\alpha_{k}}}^{j-1} \psi_{v} \\
& =S_{M_{\alpha_{k}}} f+\lambda_{k}\left(D_{j}-D_{M_{\alpha_{k}}}\right) \\
& =S_{M_{\alpha_{k}}} f+\lambda_{k} \psi_{M_{\alpha_{k}}} D_{j-M_{\alpha_{k}}} \\
& =I_{1}+I_{2} .
\end{aligned}
$$


In view of (1) we can write that

$$
\left\|I_{1}\right\|_{1} \leq\left\|S_{M_{\alpha_{k}}} f\right\|_{1} \leq c\|f\|_{H_{1}} .
$$

By combining (8) and (14) we get that

$$
\left\|S_{n} f\right\|_{1} \geq\left\|I_{2}\right\|_{1}-\left\|I_{1}\right\|_{1} \geq \lambda_{k} L\left(n-M_{\alpha_{k}}\right)-c\|f\|_{H_{1}} .
$$

Hence,

$$
\begin{aligned}
& \sup _{n \in \mathbb{N}_{+}} \frac{1}{n \varphi_{n}} \sum_{k=1}^{n}\left\|S_{k} f\right\|_{1} \\
\geq & \frac{1}{2 M_{\alpha_{k}} \varphi_{2 M_{\alpha_{k}}}} \sum_{\left\{M_{\alpha_{k}} \leq l \leq 2 M_{\alpha_{k}}\right\}}\left\|S_{l} f\right\|_{1} \\
\geq & \frac{1}{2 M_{\alpha_{k}} \varphi_{2 M_{\alpha_{k}}}} \sum_{\left\{M_{\alpha_{k}} \leq l \leq 2 M_{\alpha_{k}}\right\}}\left(\frac{L\left(l-M_{\alpha_{k}}\right) \varphi_{2 M \alpha_{k}}^{1 / 2}}{\log ^{1 / 2} M_{\alpha_{k}}}-c\|f\|_{H_{1}}\right) \\
\geq & \frac{c \varphi_{2 M \alpha_{k}}^{1 / 2}-1}{2 M_{\alpha_{k}} \log ^{1 / 2} M_{\alpha_{k}} \varphi_{2 M_{\alpha_{k}}}} \sum_{l=1}^{l^{1 / 2}} L(l)-c\|f\|_{H_{1}}^{1 / 2} \\
\geq & \frac{c \varphi_{2 M \alpha_{k}}^{1 / 2} \log M_{\alpha_{k}}}{\log ^{1 / 2} M_{\alpha_{k}} \varphi_{2 M_{\alpha_{k}}}} \geq \frac{c \log ^{1 / 2} M_{\alpha_{k}}}{\varphi_{2 M_{\alpha_{k}}}^{1 / 2}} \rightarrow \infty, \text { as } k \rightarrow \infty .
\end{aligned}
$$

The proof is complete.

Acknowledgment: The author would like to thank the referee for helpful suggestions, which improved the final version of the paper.

\section{REFERENCES}

[1] G. N. AGAEV, N. Ya. VILENKIN, G. M. DZHAFARLY and A. I. RUBINSHTEIN, Multiplicative systems of functions and harmonic analysis on zero-dimensional groups, Baku, Ehim, 1981 (in Russian).

[2] I. BLAHOTA, K. NAGY, L. E. PERSSON, G. TEPHNADZE, A sharp boundedness result concerning some maximal operators of partial sums with respect to Vilenkin systems, Georgian Math., J., (to appear).

[3] I. BLAhOTA, L. E. PERSSON, G. TEPHNADZE, Two-sided estimates of the Lebesgue constants with respect to Vilenkin systems and applications, Glasgow Math. J., doi: 10.1017/S0017089516000549.

[4] R.R. Coifman, G. L. Weiss, Extentions of Hardy spaces and their use in analysis, Bull. Amer. Math. Acad. Sinica, 83 (1977), 569-645.

[5] S. FRIDLI and P. SIMON, On the Dirichlet kernels and Hardy space with resect to the Vilenkin system, Acta Math. Hung., 45 (1-2) 1985, 223-234.

[6] N. J. FUJII, A maximal inequality for $H_{1}$ functions on the generalized Walsh-Paley group, Proc. Amer. Math. Soc. 77 (1979), lll-116.

[7] G. GÂT, Investigations of certain operators with respect to the Vilenkin system, Acta Math. Hung., 61 (1993), 131-149. 
[8] B. I. GOLUBOV, A. V. EFIMOV and V. A. SKVORTSOV, Walsh series and transforms, (Russian) Nauka, Moscow, 1987, English transl: Mathematics and its Applications, 64. Kluwer Academic Publishers Group, Dordrecht, 1991.

[9] S. F. LUKOMSKII, Lebesgue constants for characters of the compact zerodimensional abelian group, East J. Approx. 15 (2009), no. 2, 219-231.

[10] I. MEZO and P. SIMON Integrals of weighted maximal kernels with respect to Vilenkin systems, Publ. Math. Debrecen, no.: 3580 (2007), 1-9.

[11] N. MEMIĆ, I. SIMON and G. TEPHNADZE, Strong convergence of two-dimensional Vilenkin-Fourier series, Math. Nachr., 289, 4 (2016) 485-500.

[12] L. E. PERSSON, G. TEPHNADZE, P. WALL, On an approximation of twodimensional Walsh-Fourier series in the martingale Hardy spaces, Ann. Funct. Anal., 9,1 (2018), 137-150.

[13] L. E. PERSSON, G. TEPHNADZE, P. WALL, On the maximal operators of VilenkinNörlund means, J. Fourier Anal. Appl., 21, 1 (2015), 76-94.

[14] F. SCHIPP, W. R. WADE, P. SIMON and J. PÁL, Walsh series. An introduction to dyadic harmonic analysis, Adam Hilger, Ltd., Bristol, 1990.

[15] F. SCHIPP, Certain rearrangements of series in the Walsh series, Mat. Zametki, 18 (1975), 193-201.

[16] P. SIMON, Inverstigations wish respect to the Vilenkin sistem, Annales Univ. Sci. Budapest Eotv., Sect. Math., 28 (1985) 87-101.

[17] P. SIMON, Strong convergence of certain means with respect to the Walsh-Fourier series, Acta Math. Hung., 49 (1-2) (1987), 425-431.

[18] P. SIMON, Strong Convergence Theorem for Vilenkin-Fourier Series, J. Math. Anal. Appl., 245, (2000), pp. 52-68.

[19] P. SIMON, $\left(L^{1}, H\right)$-type estimations for some operators with respect to the Walsh?Paley system, Acta Math. Hung., 46 (3-4) (1985), 307-310.

[20] B. SMITH, A strong convergence theorem for $H_{1}(T)$, in Lecture Notes in Math., 995, Springer, Berlin, 1994, 169-173.

[21] G. TEPHNADZE, On the partial sums of Walsh-Fourier series, Colloq. Math., 141, 2 (2015), 227-242.

[22] G. TEPHNADZE, A note on the norm convergence by Vilenkin-Fejér means, Georgian Math. J., 21, 4 (2014), 511-517.

[23] G. TEPHNADZE, On the partial sums of Vilenkin-Fourier series, J. Contemp. Math. Anal., 49, 1 (2014), 23-32.

[24] G. TEPHNADZE, Strong convergence of two-dimensional Walsh-Fourier series, Ukr. Math. J., 65, 6 (2013), 822-834.

[25] N. Ya. VILENKIN, On a class of complete orthonormal systems, Izv. Akad. Nauk. U.S.S.R., Ser. Mat., 11 (1947), 363-400.

[26] F. WEISZ, Martingale Hardy spaces and their applications in Fourier Analysis, Springer, Berlin-Heideiberg-New York, 1994.

[27] F. WEISZ, Summability of multi-dimensional Fourier series and Hardy spaces, Mathematics and its Applications, 541. Kluwer Academic Publishers, Dordrecht, 2002.

G.Tutberidze, The University of Georgia, school of Informatics, Engineering and Mathematics, IV, 77a Merab Kostava St, Tbilisi, 0128, Georgia, \& Department of Engineering Sciences and Mathematics, LuleßUniversity of Technology, SE-971 87 LuleA, Sweden.

E-mail address: giorgi.tutberidze1991@gmail.com 